Case report

Perinatology Vol. 31, No. 2, June, 2020 https://doi.org/10.14734/PN.2020.31.2.85

\author{
Jung Eun Park, MD, \\ Ki-Jin Ryu, MD, \\ Seung Hyun Nam, MD, \\ Geum Joon Cho, MD, PhD, \\ Soon-Cheol Hong, MD, PhD, \\ Min-Jeong Oh, MD, PhD, \\ Hai-Joong Kim, MD, PhD, \\ Ki Hoon Ahn, MD, PhD
}

Department of Obstetrics and Gynecology, Korea University College of Medicine, Seoul, Korea

Received: 18 November 2019

Revised: 11 December 2019

Accepted: 17 December 2019

Correspondence to

Ki Hoon Ahn, MD, PhD

Department of Obstetrics and

Gynecology, Korea University College

of Medicine, 73 Goryeodae-ro,

Seongbuk-gu, Seoul 02841, Korea

Tel: +82-2-920-6777

Fax: +82-2-921-5357

E-mail:akh1220@hanmail.net

Copyright@ 2020 by The Korean Society of Perinatology

This is an Open Access article distributed under the terms of the Creative Commons Attribution Non-Commercial License (http://creativecommons.org/ license/by-nc/4.0/), which permits unrestricted non-commercial use, distribution, and reproduction in any medium, provided that the original work is properly cited.

\section{Trisomy 12p Syndrome Presenting with Fetal Growth Restriction and Polyhydramnios}

\begin{abstract}
A 39-year-old nulliparous woman was referred at $36+6$ weeks gestational age, and a prenatal fetal ultrasonography examination found fetal growth restriction $(2,190 \mathrm{~g}<5 \%)$, polyhydramnios (amniotic fluid index: $31.76 \mathrm{~cm}$ ), and absent end-diastolic flow in the umbilical artery. A male baby was born, and fetal dysmorphism including round face, long philtrum, low-set ears, chest retraction, edematous hands and feet, and a simian line on both hands were identified. He was diagnosed with trisomy 12p [der(18)t(12;18)(p11.2;p11.31)] by chromosomal study. The father's chromosomes appeared normal; however, the mother's chromosomes had a balanced translocation identified as $\mathrm{t}(12 ; 18)(\mathrm{p} 11.2 ;$ p11.31). Patent ductus arteriosus, germinal matrix hemorrhage, and hemivertebrae were diagnosed, and respiratory distress, hyperinsulinemic hypoglycemia, and hypothyroidism were also detected. The combination of fetal growth restriction and polyhydramnios may be a significant sign of chromosomal abnormalities, and targeted sonography and a chromosomal analysis should be offered prenatally in such cases.
\end{abstract}

Key Words: Chromosome aberrations, Fetal growth retardation, Polyhydramnios, Chromosome 12, 12 p trisomy

\section{Introduction}

Fetal growth restriction (FGR) is a common complication of pregnancy associated with a variety of adverse perinatal outcomes, and is frequently accompanied by oligohydramnios but rarely by polyhydramnios. FGR combined with polyhydramnios has been revealed to be associated with increased perinatal mortality, maternal complications, and suspicion of chromosomal abnormality or fetal anomaly. ${ }^{1}$ Here, we report a case of a fetus complicated with FGR and polyhydramnios, who was finally diagnosed with partial trisomy 12p, a rare chromosomal anomaly.

\section{Case}

A 39-year-old nulliparous woman was referred to Korea University Anam Hospital at 36+ 6 weeks gestational age (GA) for FGR. She had taken medicines for epilepsy for 6 years. The previous antenatal screening tests had revealed FGR from 27+4 GA, and there was no other record of abnormalities on level II ultrasonography at 22 GA. Our fetal ultrasonography

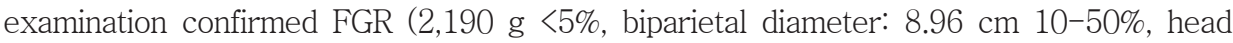

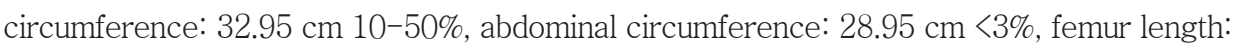
$5.89 \mathrm{~cm}<3 \%$ ), polyhydramnios (amniotic fluid index: $31.76 \mathrm{~cm}$ ), and absent or reversed enddiastolic flow in the umbilical artery (Fig. 1). A male baby was born by emergency cesarean section. The neonate weighed 2,260 g (3rd to 10th), and we identified fetal dysmorphism, including round face, long philtrum, low-set ears, chest retraction, edematous hands and feet, 


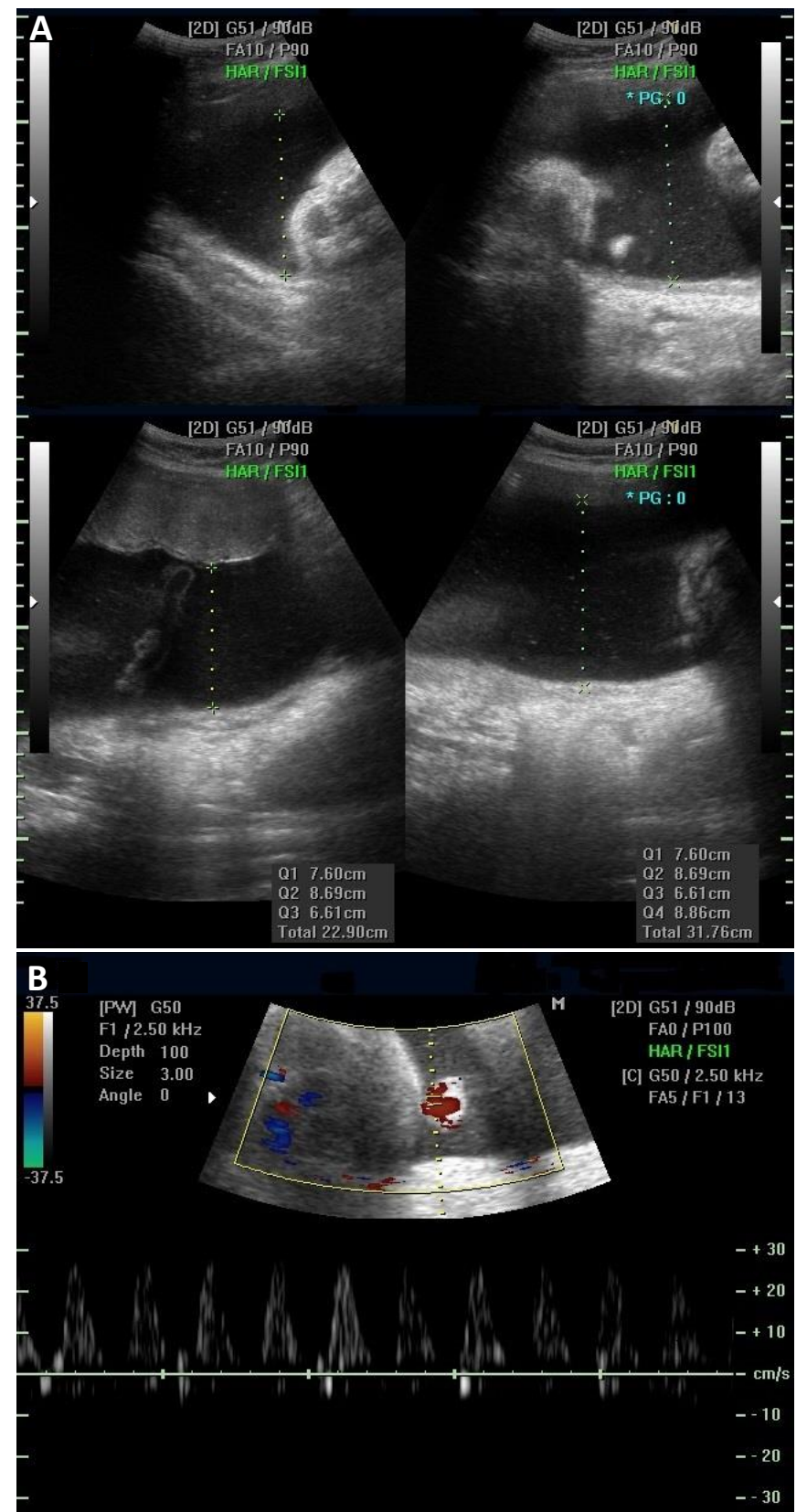

Fig. 1. Abnormal findings on fetal ultrasonography examination at 36+6 weeks gestational age. (A) Polyhydramnios (AFI: $31.76 \mathrm{~cm}$ ). (B) Absent or reversed end-diastolic flow in the umbilical artery. AFI, amniotic fluid index.

and a simian line on both hands (Fig. 2). He cried and sucked weakly, and had reduced movements.

This baby was diagnosed as having partial trisomy 12p [der(18)t(12;18)(p11.2;p11.31)] by chromosomal study (Fig. 3). Patent ductus arteriosus, germinal matrix hemorrhage, and hemivertebrae were diagnosed on neonatal examinations (Fig.

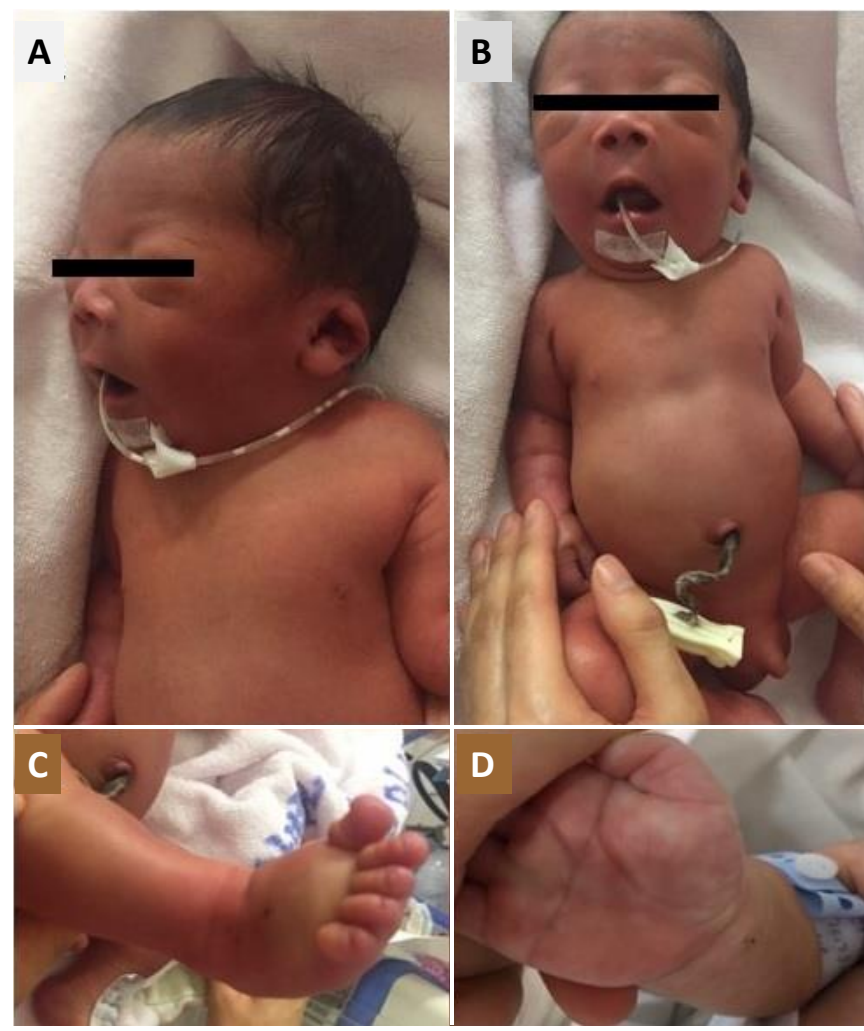

Fig. 2. Morphology of the newborn. (A, B) Round face, long philtrum, low-set ears, chest retraction. (C) Edematous foot. (D) Simian line on hand.

4). In addition, other medical issues such as respiratory distress, hyperinsulinemic hypoglycemia (serum glucose: 29), and hypothyroidism (thyroid stimulating hormone: $41.5 \mathrm{uIU} / \mathrm{mL}$, free T4: $1.20 \mathrm{ng} / \mathrm{dL}$ ) were also detected. The parents of this baby underwent chromosomal analyses. The father's chromosomes appeared normal; however, the mother's chromosomes had a balanced translocation identified as t(12;18)(p11.2;p11.31).

\section{Discussion}

Chromosomal anomalies are one of the most common causes of various hereditary malformations and mental retardations. Fetuses with trisomy 21, 18, and 13 have been known to survive pregnancy until birth; most other trisomy fetuses are spontaneously aborted in the first trimester. Trisomy 12p syndrome is a rare chromosomal abnormality, which presents with craniofacial dysmorphism, developmental or growth delay, psychomotor retardation, and generalized hypotonia. ${ }^{2}$ Since 

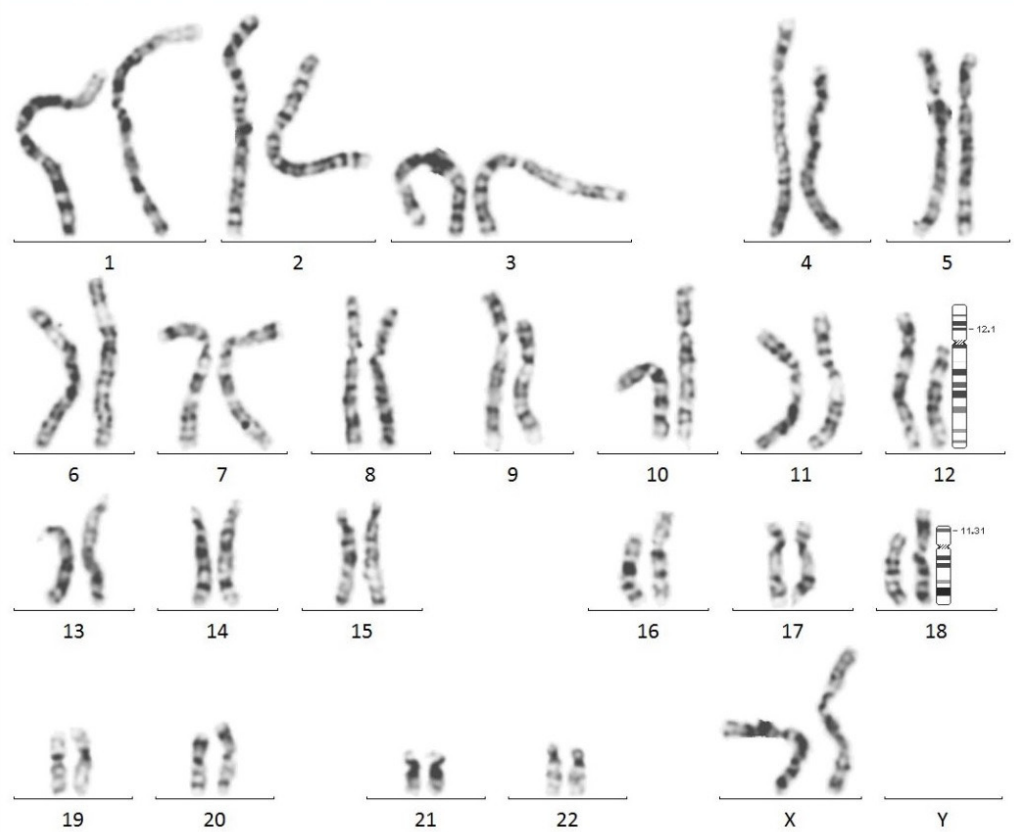

Fig. 3. Partial trisomy 12p [der(18)t(12;18)(p11.2;p11.31)] diagnosed by chromosomal study.

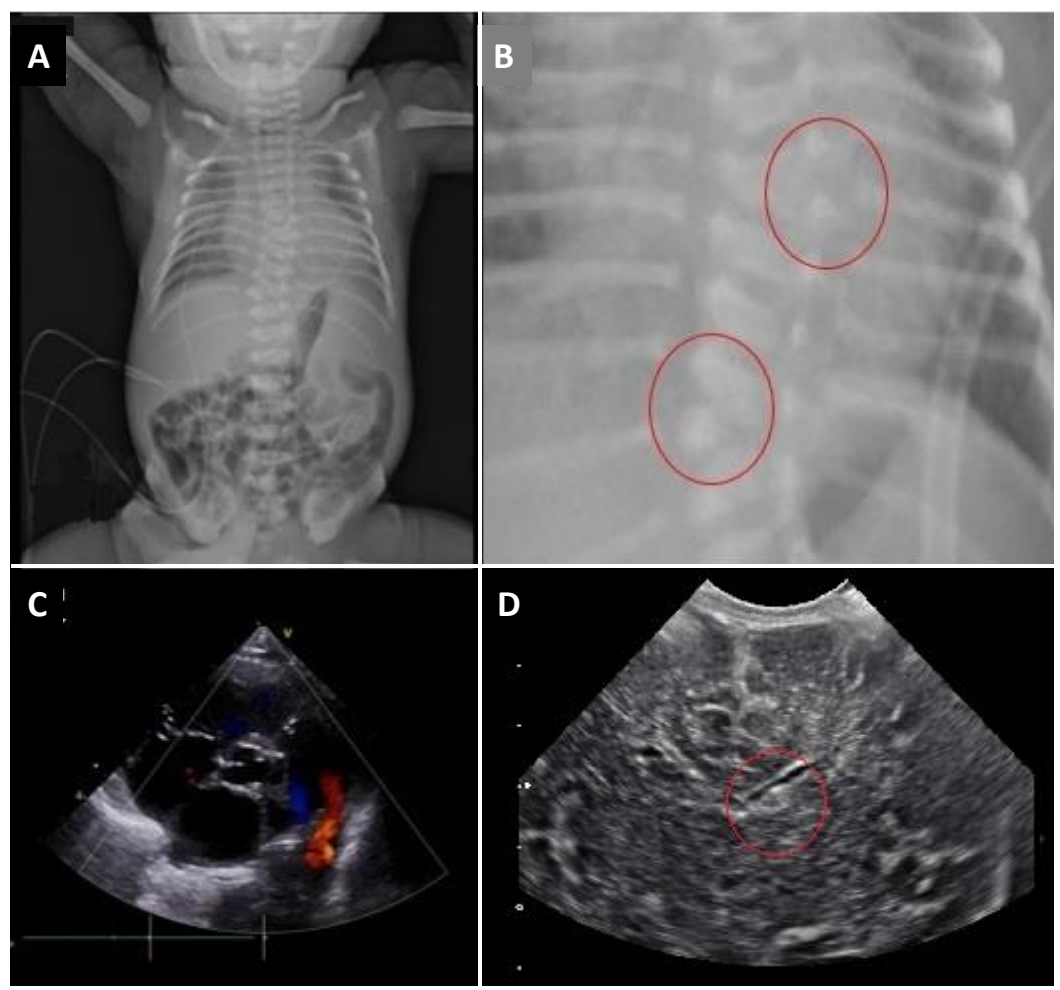

Fig. 4. Radiologic and sonographic imaging findings of the newborn. (A) Hemivertebrae. (B) A lack of formation of one half of vertebral body (red circles). (C) Patent ductus arteriosus, $3 \mathrm{~mm}$, bidirectional. (D) 4-mm sized hyperechoic lesion in left caudothalamic groove, grade 1 germinal matrix hemorrhage(red circle). 
the first case with trisomy 12 p was described in $1973,{ }^{3}$ only approximately 30 cases of trisomy 12 p have been reported, and the majority of them resulted from malsegregation of a balanced parental translocation. ${ }^{4,5}$

Previously reported cases of trisomy of the short arm of chromosome 12 have shown overlapped pathognomonic craniofacial features, referred to as 12p syndrome, as the following: flat, round, long face with prominent cheeks, or malar hypoplasia; prominent forehead with frontal receding hair line; hypertelorism; epicanthus; short palpebral fissure; broad, flat nasal bridge; short nose with anteverted nares; low set ears; long philtrum; thin upper lip vermillion; everted, prominent lower lip; prominent chin; underbite, peg teeth; missing permanent teeth; bruxism; bifid incisor; short neck; delayed developmental milestones; and hypotonia. ${ }^{6}$ In this case, common characteristics of trisomy 12p such as round face, long philtrum, low-set ears, hypertelorism, wide and flat bridge of nose, and anteverted nares were found, in addition to chest retraction, hemivertebrae, edematous hands and feet, and a simian line on both hands. Although the craniofacial dysmorphism of trisomy 12p has been described well, the antenatal sonographic findings such as the FGR with polyhydramios and absent or reversed end-diastolic flow in the umbilical artery seen in this case, have not been reported.

The combination of polyhydramnios and FGR is known to be an ominous antenatal sign and an independent risk factor for perinatal mortality. ${ }^{1}$ In a previous report, the majority of fetuses with those antenatal findings had major anomalies or chromosome abnormalities, or both, even when other sonographic abnormalities were absent: trisomy 18 was the most common aneuploidy among them. ${ }^{7}$ Some authors suggested that those findings, along with ventriculomegaly, are important prenatal ultrasonography markers of Miller-Dieker lissencephaly syndrome, associated with monosomy $17 \mathrm{p} .^{8}$ For trisomy $12 \mathrm{p}$, although the craniofacial features of this defect are well described, information on antenatal findings including the amniotic fluid status is scarce, except for one case report of a trisomy 12p fetus with FGR and oligohydramnios that resulted in neonatal death. ${ }^{9}$ The clinical presentation of trisomy 12p can be varied, depending on the trisomy chromosome length, presence of other chromosomal anomalies, and the presence of mosaicism, ${ }^{4}$ and the effect of this defect on the amniotic fluid status should be investigated with more studies. In this case, FGR with polyhydramnios and absent end-diastolic flow in the umbilical artery were the only findings that were prenatally detected. Although the accuracy of prenatal ultrasonography has been greatly improved in recent decades, fetal craniofacial dysmorphism and other structural anomalies can be missed on routine examination. Given these findings and the known poor prognosis of FGR with polyhydramnios, chromosome analysis and detailed fetal evaluation should be offered when polyhydramnios and FGR are identified prenatally.

In conclusion, the combination of FGR and polyhydramnios may be a significant sign of congenital anomalies and chromosomal abnormalities. Targeted sonography and a chromosomal analysis should be offered prenatally in such cases.

\section{Conflict of interest}

No potential conflict of interest relevant to this article was reported.

\section{References}

1) Erez O, Shoham-Vardi I, Sheiner E, Dukler D, Bashiri A, Mazor M. Hydramnios and small for gestational age are independent risk factors for neonatal mortality and maternal morbidity. Arch Gynecol Obstet 2005;271:296-301.

2) Hong B, Zunich J, Openshaw A, Toydemir RM. Clinical features of trisomy 12 mosaicism-Report and review. Am J Med Genet A 2017;173: 1681-6.

3) Uchida IA, Lin CC. Identification of partial 12 trisomy by quinacrine fluorescence. J Pediatr 1973;82:269-72.

4) Segel R, Peter I, Demmer LA, Cowan JM, Hoffman JD, Bianchi DW. The natural history of trisomy 12p. Am J Med Genet A 2006;140:695-703.

5) Kang JU, Park KY, Lee OK. A case of trisomy $12 p$ due to paternal 12;18 translocation, t(12:18)(p12.1:p11.31). J Korean Soc Neonatol 2006;13: 154-9.

6) Roberts W, Zurada A, Zurada-ZieliŃSka A, Gielecki J, Loukas M. Anatomy of trisomy 12. Clin Anat 2016;29:633-7.

7) Sickler GK, Nyberg DA, Sohaey R, Luthy DA. Polyhydramnios and fetal intrauterine growth restriction: ominous combination. J Ultrasound Med 1997;16:609-14.

8) Lin CY, Chen CP, Liau CL, Su PH, Tsao TF, Chang TY, et al. Prenatal diagnosis of monosomy 17p (17p13.3-->pter) associated with polyhydramnios, intrauterine growth restriction, ventriculomegaly, and Miller- 
Dieker lissencephaly syndrome in a fetus. Taiwan J Obstet Gynecol 2009;48:408-11.
9) Bischoff FZ, Zenger-Hain J, Moses D, Van Dyke DL, Shaffer LG. Mosaicism for trisomy 12: four cases with varying outcomes. Prenat Diagn 1995;15: 1017-26 